\title{
Measurement of $\mathbf{W}$ Boson Helicity Fractions in $t \bar{t}$ Decays at DZero and CDF
}

\author{
Chris Potter ${ }^{1}$ \\ 1- McGill University - Physics Department \\ Montreal - Canada
}

\begin{abstract}
The properties of the $\mathrm{W}$ boson can be probed in top quark decays $t \rightarrow b W$. The CDF and DZero collaborations have released updated measurements of the $W$ boson helicity fractions $f_{0}, f_{+}$and $f_{-}$. The analysis techniques and updated measurements are discussed. This paper summarizes the talk [1] given at the DIS2008 conference.
\end{abstract}

\section{Phenomenology Overview}

In the Standard Model (SM), the top quark decays almost exclusively to a bottom quark and a $W$ boson. These decays provide an opportunity to probe the structure of the $t b W$ vertex. The generic Lagrangian describing the $t b W$ vertex [2] is:

$$
\mathcal{L}_{t b W}=\frac{g}{\sqrt{2}} W_{\mu}^{-} \bar{b} \gamma^{\mu}\left(f_{1}^{L} P_{L}+f_{1}^{R} P_{R}\right) t-\frac{g}{\sqrt{2} m_{W}} \partial_{\nu} W_{\mu}^{-} \bar{b} \sigma^{\mu \nu}\left(f_{2}^{L} P_{L}+f_{2}^{R} P_{R}\right) t
$$

The $W$ helicity fractions $f_{-}$(left-handed), $f_{+}$(right-handed) and $f_{0}$ (longitudinal) are defined by

$$
\begin{gathered}
f_{0}=a_{t}^{2}\left(1+x_{0}\right) /\left[a_{t}^{2}\left(1+x_{0}\right)+2\left(1+x_{m}+x_{p}\right)\right] \\
f_{-}=2\left(1+x_{m}\right) /\left[a_{t}^{2}\left(1+x_{0}\right)+2\left(1+x_{m}+x_{p}\right)\right] \\
f_{+}=2 x_{p} /\left[a_{t}^{2}\left(1+x_{0}\right)+2\left(1+x_{m}+x_{p}\right)\right]
\end{gathered}
$$

where $a_{t}=m_{t} / m_{W}$ and $x_{0}, x_{p}$ and $x_{m}$ are simple functions of the $f_{1,2}^{L, R}[2]$. In $t \rightarrow b W \rightarrow$ $b \ell \nu$ events, if $\cos \theta^{\star} \equiv c$ (Fig. 1) is the cosine of the angle between the charged lepton from the $W$ decay and the top in the $W$ rest frame, then the distribution of $\mathrm{c}$ is given by $f_{+}(1+c)^{2}+2 f_{0}\left(1-c^{2}\right)+f_{-}(1-c)^{2}$. In the case of hadronic $W$ decay, the down-type quark takes the role of the charged lepton in the definition of $\cos \theta^{\star}$.

\begin{tabular}{|c|c|c|c|}
\hline Fraction & SM Prediction & MSSM & Technicolor \\
\hline$f_{+}$ & $3.4 \times 10^{-4}$ & - & - \\
$f_{0}$ & $\frac{m_{t}^{2}}{2 m_{W}^{2}+m_{t}^{2}} \approx 0.703$ & 0.699 & 0.707 \\
$f_{-}$ & $1-f_{0}-f_{+} \approx 0.297$ & 0.301 & 0.293 \\
\hline
\end{tabular}

Table 1: Helicity fractions predicted in the SM, MSSM and Technicolor [2].

Different theoretical models predict different helicity fractions. See Table 1 for the helicity fractions predicted in the SM, the Minimal Supersymmetric Model (MSSM) and Technicolor [2]. See Fig. 1 for the $\cos \theta^{\star}$ distribution predicted by the SM.

DIS 2008 

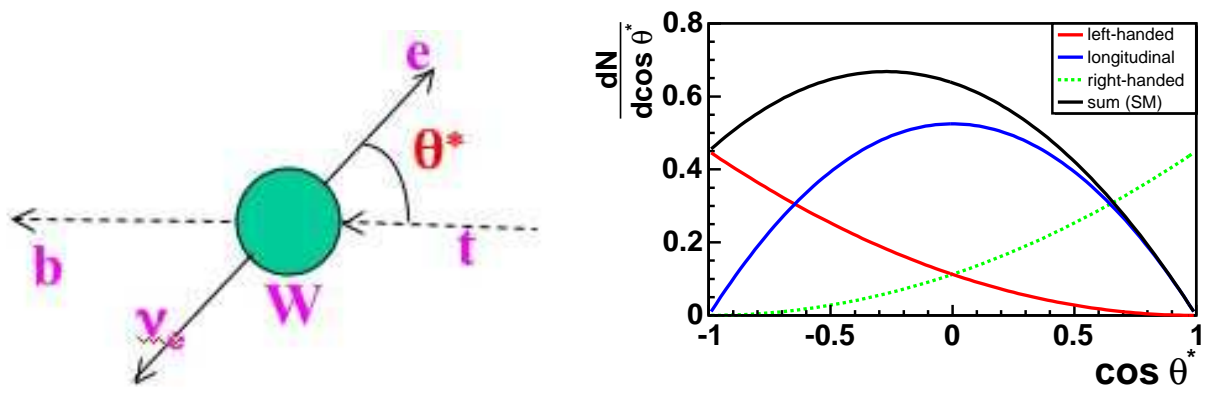

Figure 1: At left, the definition of the helicity angle $\theta^{\star}$ in the decay $t \rightarrow b W \rightarrow b \ell \nu$. At right, the distributions of $\cos \theta^{\star}$ for pure left-handed, pure right-handed, pure longitudinal samples, and the SM.

\section{Recent Tevatron Analyses}

Two experiments at the Tevatron have recently reported updated measurements of the $W$ boson helicity fractions. DZero has a single analysis (Ref. [3]) using a $1 \mathrm{fb}^{-1}$ dataset, and in addition to the lepton-plus-jets channel it also exploits the dilepton channel. CDF has three independent analyses (Ref. $[4,5,6]$ which all use a $1.9 \mathrm{fb}^{-1}$ dataset to measure the $W$ boson helicity in the lepton-plus-jets channel.

DZero Analysis. In the DZero analysis, a discriminant is calculated from kinematics and jet flavor: signal event jets and leptons have higher $p_{T}$, are more central, and contain two b-jets. The four-vectors of the $W$ bosons and top quarks are reconstructed and $\cos \theta^{\star}$ is calculated. Templates for signal and background with varying helicity states $-, 0,+$ are created. Helicity fractions $f_{0}$ and $f_{+}$are extracted from a binned Poisson likelihood $L\left(f_{0}, f_{+}\right)$. The hadronic $\mathrm{W}$ decay in the $\ell+$ jets channel gives an ambiguity in the sign of $\cos \theta^{\star}$, but $\left|\cos \theta^{\star}\right|$ improves the $f_{0}$ statistical error by $20 \%$. The main backgrounds are $W+$ jets and multijets for the $\ell+$ jets channels and $W W+$ jets and $Z+$ jets for the dilepton channel.

CDF Unfolding Method. The CDF unfolding method analysis uses $\ell+$ jets channel only, and the main backgrounds are $W+$ jets and dijets. A quantity $\Psi=P_{\nu} P_{b-\text { light }} \chi^{2}$ is calculated to suppress background and resolve signal combinatoric ambiguity. $P_{\nu}$ is a weighting factor for $p_{z}^{\nu}, P_{b-l i g h t}$ is a measure of similarity with light jets, and

$$
\chi^{2}=\frac{\left(m_{W \rightarrow j j}-M_{W \rightarrow j j}\right)^{2}}{\sigma_{M_{W \rightarrow j j}}^{2}}+\frac{\left(m_{t o p \rightarrow b \ell \nu}-m_{t o p \rightarrow b j j}\right)^{2}}{\sigma_{\Delta M_{t}}^{2}}+\frac{\left(P_{\text {energy }}-\alpha\right)^{2}}{\sigma_{\text {Penergy }}^{2}}
$$

To extract $f_{+}$and $f_{0}$ a binned likelihood $L\left(f_{0}, f_{+}\right)$is constructed to find the values which maximize the probability for the given data using signal and background templates $(-, 0,+)$.

CDF Matrix Element Method. In the CDF matrix element analysis, background is suppressed by requiring isolated high $E_{T}$ lepton ( $e$ or $\mu$ ), large $E_{T}^{\text {miss }}$, four or more high $E_{T}$ jets, tight secondary vertex requirements, and scalar sum of all transverse energy in the event $H_{T}>200 \mathrm{GeV}$. This analysis suffers background mainly from $W+$ jets and QCD 

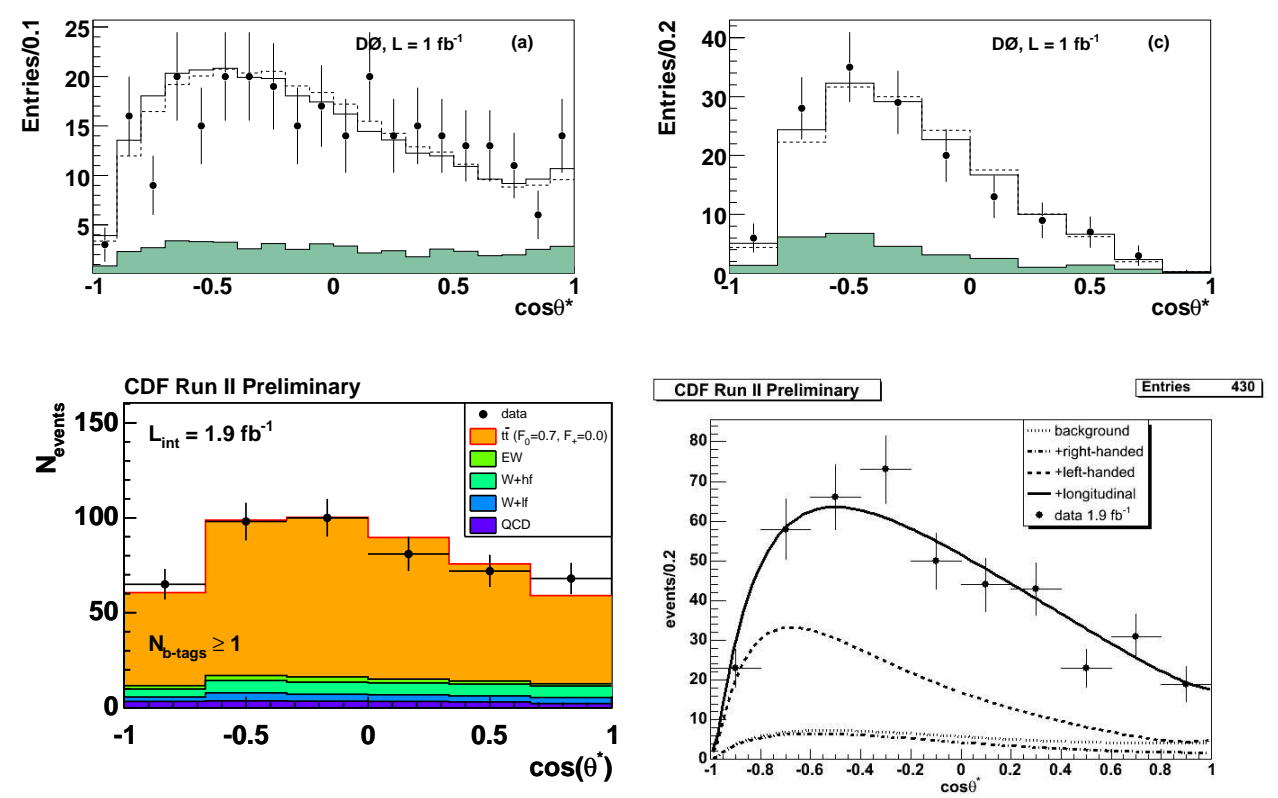

Figure 2: Reconstructed $\cos \theta^{\star}$ in the DZero $\ell+$ jets channel (top left) and in the DZero dilepton channel (top right). Reconstructed $\cos \theta^{\star}$ in the $\operatorname{CDF} \ell+$ jets channel with the unfolding method (bottom left) and CDF template method (bottom right).

dijets. The $f_{+}$parameter is set to zero and matrix elements are employed to generate a likelihood $L\left(f_{0}, C_{s}\right)$ for $N$ events:

$$
L\left(f_{0}, C_{s}\right)=\prod_{i=1}^{N} C_{s} P_{t \bar{t}}\left(\vec{x}_{i} ; f_{0}\right)+\left(1-C_{s}\right) P_{W+j e t s}\left(\vec{x}_{i}\right)
$$

where $C_{s}$ is the fraction of signal events. Then $f_{0}$ is extracted from the likelihood. The probabilities $P(\vec{x})$ are derived for signal $t \bar{t}$ and background $(W+$ jets) by evaluating the leading order matrix elements at the measured vectors $\vec{x}$ :

$$
P(\vec{x})=\frac{1}{\sigma_{o b s}} \int \frac{d \sigma(\vec{y})}{d \vec{y}} f\left(\tilde{q}_{1}\right) f\left(\tilde{q}_{2}\right) W(\vec{x}, \vec{y}) d \tilde{q}_{1} d \tilde{q}_{2} d \tilde{p}_{t \bar{t}}^{x} d \tilde{p}_{t \bar{t}}^{y} d \vec{y}
$$

where $f$ are the parton density functions, $W(\vec{x}, \vec{y})$ is a transfer function describing the probability that parton with $\vec{y}$ will be measured as $\vec{x}$. As a crosscheck, the $e$ and $\mu$ channels are evaluated separately. In the electron channel, $f_{0}=0.660 \pm 0.111$ (stat) while in the muon channel $f_{0}=0.609 \pm 0.128$ (stat).

CDF Template Method. The CDF template method analysis uses the $\ell+$ jets channel and suffers background mainly from $W+$ jets. Background is suppressed by requiring one $e$ or $\mu, E_{T}^{m i s s}$, scalar $H_{T}>250 \mathrm{GeV}$, four or more jets, one of which is identified as a b-jet by a secondary vertex tagger. The $\cos \theta^{\star}$ distribution is fit with a $3 \mathrm{rd}$ degree polynomial times two exponentials. An unbinned extended likelihood fitter is used to extract $f_{+}$and $f_{0}$ with the likelihood:

$$
\mathcal{L}=G\left(b \mid \mu_{b}, \sigma_{b}\right) \times P\left(s+b \mid \mu_{s}+\mu_{b}\right) \times \prod_{i=1}^{N}\left[f_{b} p_{b}(c)+\left(1-f_{b}\right) p_{s}(c)\right] .
$$




\begin{tabular}{|c|c|c|c|c|}
\hline Experiment & Channel & $\mathcal{L}$ & $f_{+}$ & $f_{0}$ \\
\hline DZero & $\ell+$ jets & $1 \mathrm{fb}^{-1}$ & $0.119 \pm 0.090 \pm 0.053$ & $0.425 \pm 0.166 \pm 0.102$ \\
& dilepton & & $-0.002 \pm 0.047 \pm 0.047$ & $0.619 \pm 0.090 \pm 0.052$ \\
\hline CDF UM & $\ell+$ jets & $1.9 \mathrm{fb}^{-1}$ & $0.15 \pm 0.10 \pm 0.05$ & $0.38 \pm 0.21 \pm 0.07$ \\
& & & $0.01 \pm 0.05 \pm 0.03$ & $0.66 \pm 0.10 \pm 0.06$ \\
\hline CDF ME & $\ell+$ jets & $1.9 \mathrm{fb}^{-1}$ & - & $0.637 \pm 0.084 \pm 0.069$ \\
\hline CDF TM & $\ell+$ jets & $1.9 \mathrm{fb}^{-1}$ & $-0.03 \pm 0.07 \pm 0.03$ & $0.65 \pm 0.19 \pm 0.03$ \\
& & & $-0.04 \pm 0.04 \pm 0.03$ & $0.59 \pm 0.11 \pm 0.04$ \\
\hline
\end{tabular}

Table 2: Recent measurements of the $W$ boson helicity fractions from DZero and CDF unfolding method (UM), CDF matrix element method (ME) and CDF template method (TM). When two results are reported, the top number results from a simultaneous determination of $f_{+}$and $f_{0}$ while the bottom number results from fixing $f_{+}=0$ for $f_{0}$ and $f_{0}=0.7$ for $f_{+}$.

\section{Conclusion}

See Table 2 for the results of the recent Tevatron measurements. The results have not yet been combined. All measurements are consistent with the SM. Systematic errors are at a level such that the measurements are not presently able to discriminate between SM, MSSM and Technicolor predictions, which differ at the percent level.

As the Tevatron accumulates luminosity, the statistical errors will become smaller than the systematic uncertainties and the analyses will become systematics limited. Uncertainties are evaluated in ensemble tests in which the source of systematic uncertainty is sampled repeatedly, the helicity fractions recalculated. The mean difference gives the uncertainty.

The overall systematic uncertainties on $f_{0}$ are $16 \%$ (DZero), $11 \%$ (CDF matrix element), $10 \%$ (CDF unfolding method), and 5\% (CDF template method). The largest single systematic uncertainties reported are due to the event generator $(11 \%$ DZero, $8 \%$ CDF matrix element). These are further broken down into uncertainties due to final state radiation, initial state radiaqtion, and parton density functions in the remaining analyses.

\section{Acknowledgments}

Grateful acknowledgment is made to the DZero and CDF collaborations for assistance in writing this summary, and in particular for providing plots and diagrams.

\section{References}

[1] Slides: http: //indico. cern. ch/materialDisplay py?contribId=116\&sessionId=15\&materialId=slides\&conf Id=24657

[2] C.-R. Chen, F. Larios, C.-P. Yuan, arXiv:hep-ph/0503040v3 (2008).

[3] DZero Collaboration, Phys. Rev. Lett. 100, 062004 (2008)

[4] http://www-cdf.fnal.gov/physics/new/top/2007/topProp/Whel_ME/

[5] http://www-cdf.fnal.gov/physics/new/top/2007/topProp/Whel_costhTemplate2fb/

[6] http://www-cdf.fnal.gov/physics/new/top/2007/topProp/WHelicityFullReco2fb/ 